1976, he was a SIAM Visiting Lecturer. He has also been a Visiting Professor at the University of Padua, Italy. In 1982, he was appointed to the Chair of Optimization and Systems Theory at the Royal Institute of Technology.

Michele Pavon was born in Venice. Italy, on October 12. 1950. He received the "Laurea" degree in mathematics from the University of Padua. Italy, in 1974, and the Ph.D. degree in mathematics from the University of Kentucky, Lexington. in 1979.

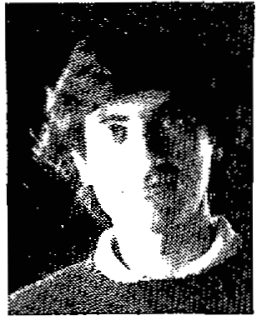

During the academic year 1974-1975, he was a Research Assistant in the Department of Mathematics, University of Padua. From March 1979 through February 1980, he served in the Italian Army. Since March 1980, he has been on the Research Staff of LADSEB-CNR, Padua, Italy. He has also held visiting positions at the University of Kentucky, Florida State University, Tallahassee, and the University of Groningen, The Netherlands. His research interests lie in the area of stochastic systems theory.

\title{
On Controllability and Observability of Time Delay Systems
}

\author{
DIETMAR SALAMON
}

\begin{abstract}
This paper deals with controllability and observability properties of time delay systems in the state space $\mathbb{R}^{n} \times L^{P}$. In particular, we prove the equivalence of spectral controllability and approximate null-controllability. Moreover, it is shown that the necessary condition for approximate $F$-controllability - obtained recently by Manitius-is also sufficient, and a verifiable and matrix type criterion for $F$-controllability is derived for systems with commensurate delays. Finally, we introduce the dual observability notion of approximate controllability and prove that the control system $\Sigma$ is exactly null-controllable if and only if the transposed delay system $\Sigma^{T}$ is continuously finally observable.
\end{abstract}

\section{INTRODUCTION}

$\mathrm{C}$ ONTROLLABILITY and observability of systems with delays in the state variables has become an area of active research in the last few years. On one hand, the algebraic systems theory, in particular that of linear systems over rings, has led to a clear connection between controllability over a ring and a spectrum assignability via feedback [12], [20], [28]. On the other hand, the functional analytic theory of infinite dimensional linear systems led to criteria for approximate controllability and observability in a function space [16]-[18], which are related to the ideas of state feedback and observers [4], [22], [24], [25]. In spite of this progress, there are still several gaps in the relations between the various concepts of controllability, stabilizability, and observability. In particular, some duality relations have not yet been clarified.

Manuscript received July 16. 1981; revised June 24. 1983. Paper recommended by E. W. Kamen, Past Chairman of the Linear Systems Committee. This work was supported by the Forschungsschwerpunkt Dynamische Systeme. University of Bremen.

The author was with the Forschungsschwerpunkt Dynamische Systeme, Universität Bremen, Bremen. West Germany. $\mathrm{He}$ is now with the Mathematics Research Center, University of Wisconsin-Madison, WI 53706.
In this paper we study linear control systems with delays in the state variables within the framework of the state space $R^{n} \times L^{p}$ $(1<p<\infty)$. The aim is to establish relationships between the exact and approximate null-controllability and certain notions of observability and to generalize and extend recent results of Manitius [17] on approximate $F$-controllability. This latter effort is motivated in part by the fact that the $F$-controllability provides via duality a clear criterion for observability of retarded systems. For more motivation of the $F$-controllability concept the reader is referred to [14] and [17].

One of the key features of this paper is the use of the structural operators $F$ and $G$ [2], [15] associated with retarded systems. These operators give a clear characterization of the structure of the semigroup operator and eliminate the burden of cumbersome notation often encountered in some work on functional differential equations. As will be seen in this paper, the use of these operators allows us to obtain very concise proofs of all the results.

Function space controllability of retarded systems has been studied via several approaches. Banks et al. [1] considered the exact controllability in $W^{1,2}$ and showed that it led to a very restrictive condition on system matrices. Pandolfi [22] has proved a criterion for feedback stabilization in the state space $\mathscr{G}$. Analogous results on spectral observability have been derived by Bhat and Koivo [3]. The null-controllability has been investigated by several Soviet authors (see, e.g., [19]) and also in [11]. Manitius and Triggiani [18] and Manitius [14], [16], [17] have characterized the approximate controllability and $F$-controllability in the product space $\mathbb{R}^{n} \times L^{2}$, and a dual concept of observability. Dynamic observers for retarded systems have been investigated by Bhat and Koivo [4], Olbrot [21], and Salamon [24], [25].

The main results of this paper are as follows. In Section II it is shown that a general retarded functional differential system is spectrally controllable if and only if it is approximately null-con- 
trollable; the latter property is dual to the final observability of a transposed system. In Section III the two necessary conditions for approximate $F$-controllability obtained recently by Manitius [17] are shown to be also sufficient. A verifiable matrix condition for $F$-controllability is then given for systems with commensurate delays. Finally, it is proved in Section IV that a retarded system is exactly null-controllable if and only if the transposed system is continuously finally observable. In Section I some preliminary results are given. In particular, it is shown that the reachable subspace of a time delay system is related to the unobservable subspace of the transposed system by means of the structural operators $F$ and $G$.

\section{Preliminaries}

\section{A. System Description and Notation}

We consider the linear control system

$$
\dot{x}(t)=L x_{t}+B_{0} u(t)
$$

where $x(t) \in \mathbb{R}^{n}, u(t) \in \mathbb{R}^{m}$, and $x_{i}(\tau)=x(t+\tau)$ for $-h \leqslant \tau \leqslant 0$ $(0<h<\infty)$. Correspondingly, $B_{0} \in \mathbb{R}^{n \times m}$ and $L$ is a (general) bounded linear operator from $\mathscr{C}=\mathscr{C}\left([-h, 0] ; \mathbb{R}^{n}\right)$ into $\mathbb{R}^{n}$, described by

$$
L_{\varphi}=\int_{-h}^{0} d \eta(\tau) \varphi(\tau), \quad \varphi \in \mathscr{C}
$$

where $\eta$ is an $n \times n$-matrix valued function of bounded variation on $R$. Without loss of generality we can assume that $\eta(\tau)=0$ for $\tau \geqslant 0, \eta(\tau)=\eta(-h)$ for $\tau \leqslant-h$, and that $\eta(\tau)$ is left continuous for $-h<\tau<0$. In particular, we consider systems with commensurate delays which means that $L$ is given by

$$
L \varphi=\sum_{j=0}^{N} A_{j} \varphi(-j \alpha), \quad \varphi \in \mathscr{C},
$$

where $\alpha=h / N>0$.

Along with $\Sigma$ we also consider the observed delay system

$$
\dot{z}(t)=L^{T} z_{t}, \quad y(t)=B_{0}^{T} z(t),
$$

where the bounded linear functional $L^{T}: \mathscr{C} \rightarrow \mathbb{R}^{n}$ is represented by the transposed matrix function $\eta^{T}(\tau)$ in an obvious manner.

It is well known [5], [6], [29] that system $\Sigma$ admits a unique solution $x(t), t \geqslant-h$, for every input $u(\cdot) \in L P_{o c}\left([0, \infty) ; \mathbb{R}^{m}\right)$ and every initial condition of the form

$$
x(0)=\varphi^{0}, \quad x(\tau)=\varphi^{1}(\tau), \quad-h \leqslant \tau<0,
$$

where $\varphi=\left(\varphi^{0}, \varphi^{1}\right) \in M^{p}=\mathbb{R}^{n} \times L^{p}\left([-h, 0] ; \mathbb{R}^{n}\right), 1<p<\infty$. The fundamental matrix solution of system $\Sigma$ will be denoted by $X(t) \in \mathbb{R}^{n \times n}, t \geqslant-h$, and corresponds to the initial condition $X(0)=I, X(\tau)=0,-h \leqslant \tau<0$, and the input $u(t) \equiv 0$. The state space $M^{p}$ of system $\Sigma$ will be endowed with the norm

$$
\|\varphi\|_{M^{p}}=\left(\left|\varphi^{0}\right|^{p}+\int_{-h}^{0}\left|\varphi^{1}(\tau)\right|^{p} d \tau\right)^{1 / p}
$$

for $\varphi \in M^{p}$ where $|\cdot|$ denotes the $p$-norm on $\mathbb{R}^{n}$. Associated with system $\Sigma$ we consider the $C_{0}$-semigroup $S(t): M^{p} \rightarrow M^{P}$ which maps every $\varphi \in M^{p}$ into the corresponding state $\left(x(t), x_{t}\right) \in M^{p}$ of $\Sigma$, (2) with $u(t) \equiv 0$. Then the evolution of system $\Sigma$ can be described by the variation-of-constants formula

$$
\left(x(t), x_{t}\right)=S(t) \varphi+\int_{0}^{t} S(t-s) B u(s) d s, \quad t \geqslant 0,
$$

where the input operator $B: \mathbb{R}^{m} \rightarrow M^{p}$ is defined by $B u=$
$\left(B_{0} u, 0\right) \in M^{p}$ for $u \in \mathbb{R}^{m}$. Correspondingly, we may define the reachable subspaces

$$
\begin{aligned}
& \mathscr{R}_{t}=\left\{\int_{0}^{t} S(t-s) B u(s) d s \mid u(\cdot) \in L^{p}\left([0, t] ; \mathbb{R}^{m}\right)\right\}, \\
& \mathscr{R}=\bigcup_{t>0} \mathscr{R}_{t} .
\end{aligned}
$$

We study the transposed system $\Sigma^{T}$ in the state space $M^{q}$, $1 / p+1 / q=1$, and denote the corresponding semigroup by $S^{T}(t)$ : $M^{q} \rightarrow M^{q}$. The output operator of system $\Sigma^{T}$ is given by $B^{*}$ : $M^{q} \rightarrow \mathbb{R}^{m}$ which maps $\psi \in M^{q}$ into $B^{*} \psi=B_{0}^{T} \psi^{0}$. Correspondingly, we may define the unobservable subspaces

$$
\begin{aligned}
& \mathscr{N}_{t}^{T}=\left\{\psi \in M^{q} \mid B^{*} S^{T}(s) \psi=0 \text { for } 0 \leqslant s \leqslant t\right\} \\
& \mathscr{N}^{T}=\bigcap_{t>0} \mathscr{N}_{t}^{T} .
\end{aligned}
$$

Sometimes we denote by $\mathscr{R}, \mathcal{N}^{T}, M^{p}$, or $W^{1, p}=W^{1, p}([-h, 0]$; $\mathbb{R}^{n}$ ) the obvious complex extension of the respective (sub)space. The infinitesimal generators of $S(t)$ and $S^{T}(t)$ will be denoted by $A$ and $A^{T}$, respectively. The spectrum of these operators can be characterized by the complex matrix function

$$
\Delta(\lambda)=\lambda I-L\left(e^{\lambda \cdot}\right)=\lambda I-\int_{-h}^{0} e^{\lambda \tau} d \eta(\tau), \quad \lambda \in \mathbb{C} .
$$

The (finite dimensional) generalized complex eigenspaces of $A$ and $A^{T}$ associated with the eigenvalue $\lambda \in \sigma(A)=\sigma\left(A^{T}\right)$ will be denoted by $X_{\lambda}$ and $X_{\lambda}^{T}$ and the corresponding canonical projection operators by $P_{\lambda}: M^{p} \rightarrow X_{\lambda}$ and $P_{\lambda}^{T}: M^{q} \rightarrow X_{\lambda}^{T}$. Moreover, we denote by $\Sigma_{\lambda}$ and $\Sigma_{\lambda}^{T}$ the finite dimensional subsystems which are obtained by projecting the systems $\Sigma$ and $\Sigma^{T}$ on the subspaces $X_{\lambda}$ and $X_{\lambda}^{T}$, respectively.

Finally, note that $M^{q}$ can be identified with the dual space of $M^{p}$ via the duality pairing

$$
\langle\psi, \varphi\rangle=\psi^{0^{T}} \varphi^{0}+\int_{-h}^{0} \psi^{1^{T}}(\tau) \varphi^{1}(\tau) d \tau
$$

for $\varphi \in M^{p}$ and $\psi \in M^{q}$. However, $S^{T}(t)$ is not the adjoint semigroup of $S(t)$. A duality relation between $S(t)$ and $S^{T}(t)$ can be given by means of the hereditary product

$$
\langle\langle\psi, \varphi\rangle\rangle=\psi^{0^{T}} \varphi^{0}+\int_{-h}^{0} \int_{\tau}^{0} \psi^{1^{T}}(\tau-\sigma) d \eta(\tau) \varphi^{1}(\sigma) d \sigma
$$

(compare [9, p. 169]). Note that this expression is well-defined for all $\varphi \in M^{p}$ and $\psi \in M^{q}$ since the convolution of an $L^{p}$ with an $L^{q}-$ function is continuous.

\section{B. The Structural Operators}

In the literature on delay systems [2], [6], [15] the structural operators $F$ and $G$ on $M^{p}$ have been introduced as follows. ${ }^{1}$

Given $\varphi \in M^{P}$, then $F \varphi \in M^{p}$ is defined by the relation

$$
\langle\psi, F \varphi\rangle=\langle\langle\psi, \varphi\rangle\rangle \quad \forall \psi \in M^{q}
$$

and $G \varphi \in M^{p}$ by

$$
\begin{aligned}
{[G \varphi]^{1}(\tau) } & =X(h+\tau) \varphi^{0}+\int_{-h}^{0} X(h+\tau+\sigma) \varphi^{1}(\sigma) d \sigma \\
{[G \varphi]^{0} } & =[G \varphi]^{1}(0) .
\end{aligned}
$$

These operators have turned out to be a very useful tool for the study of the delay systems $\Sigma$ and $\Sigma^{T}$. The main reason for this is

${ }^{1}$ The resuits in [2], [6], [15] are all in the $M^{2}$-space but can easily be extended to $M^{p}, 1<p<\infty$. 
that $F$ and $G$ satisfy the following interesting relations:

$$
F^{*} S^{T}(t)=S^{*}(t) F, \quad S^{T}(t) G^{*}=G^{*} S^{*}(t)
$$

and

$$
\begin{aligned}
S(h) & =G F, & & S^{T}(h)=G^{*} F^{*}, \\
S^{T *}(h) & =F G, & & S^{*}(h)=F^{*} G^{*} .
\end{aligned}
$$

Moreover, $[G \varphi]^{1}$ is in $W^{1, p}$ for all $\varphi \in M^{p}$, and $G$ (interpreted as an operator from $M^{p}$ into $W^{1, p}$ ) is one-to-one, onto, and continuous (see [15] or [26]). Finally, $F^{*}$ and $G^{*}$ are operators of the same type as $F$ and $G$. They are associated with the transposed equation $\dot{z}(t)=L^{T} z_{i}$ in the same manner as $F$ and $G$ are associated with the original equation $\dot{x}(t)=L x_{i}$ (see [6] and [15]).

In [16] and [17] Manitius has introduced a third operator $D$ : $L^{p}\left([-h, 0] ; \mathbb{R}^{m}\right) \rightarrow M^{p}$ in order to study controllability properties of $\Sigma$. $D \xi$ is defined by

$$
\begin{aligned}
{[D \xi]^{1}(\tau) } & =B_{0} \xi(\tau), \quad-h \leqslant \tau \leqslant 0, \\
{[D \xi]^{0} } & =0
\end{aligned}
$$

for $\xi \in L^{p}\left([-h, 0] ; \mathbb{R}^{m}\right)$. It is easy to see that the dual operator $D^{*}: M^{q} \rightarrow L^{q}\left([-h, 0] ; \mathbb{R}^{m}\right)$ is given by

$$
D^{*} \psi(\tau)=B_{0}^{T} \psi^{1}(\tau), \quad-h \leqslant \tau \leqslant 0,
$$

for $\psi \in M^{q}$.

\section{Preliminary Results}

The following result is analogous to the relations (6) and follows directly from (4) and (7).

Lemma 1.1: For $\xi \in L^{p}\left([-h, 0] ; \mathbb{R}^{m}\right)$ we have

$$
G D \xi=\int_{0}^{h} S(h-s) B \xi(-s) d s .
$$

Proof:

$$
\begin{aligned}
{[G D \xi]^{1}(\tau) } & =\int_{-h}^{0} X(h+\tau+\sigma) B_{0} \xi(\sigma) d \sigma \\
& =\int_{0}^{h} X(h+\tau-s) B_{0} \xi(-s) d s \\
& =\int_{0}^{h+\tau} X(h+\tau-s) B_{0} \xi(-s) d s \\
& =x(h+\tau), \quad-h \leqslant \tau \leqslant 0,
\end{aligned}
$$

where $x(t),-h \leqslant t \leqslant h$, is the unique solution of $\Sigma$ with control $u(t)=\xi(-t), 0 \leqslant t \leqslant h$, and zero initial condition. Hence, the statement follows from the variation-of-constants formula for the solutions of $\Sigma$ in $M^{p}$.

For every $u(\cdot) \in L^{p}\left([0, t] ; \mathbb{R}^{m}\right), t>h$, we have

$$
\begin{aligned}
\int_{0}^{t} S(t-s) B u(s) d s & \\
= & S(h) \int_{0}^{t-h} S(t-h-s) B u(s) d s \\
& +\int_{0}^{h} S(h-s) B u(t-h+s) d s .
\end{aligned}
$$

Q.E.D.

Hence, by (6) and Lemma 1.1, the following equation holds for every $t>h$ :

$$
\mathscr{R}_{t}=G F \mathscr{R}_{t-h}+\operatorname{Im} G D
$$

This leads to the following relations between $\mathscr{R}_{1}$ and $\mathscr{N}_{1}^{T}$. Lemma 1.2: Let $\psi \in M^{q}$. Then

i) $F^{*} \psi \perp \mathscr{R}_{t} \Leftrightarrow \psi \in \mathscr{N}_{t}^{T}$,

ii) for every $t>h$

$$
\psi \perp \mathscr{R}_{t} \Leftrightarrow G^{*} \psi \in \mathscr{N}_{t-h}^{T} \cap \operatorname{ker} D^{*}
$$

Proof:

i) By Fattorini [7], $F^{*} \psi$ is orthogonal to $\mathscr{R}$, if and only if

$$
B^{*} S^{*}(s) F^{*} \psi=0, \quad 0 \leqslant s \leqslant t,
$$

which, by (5), is equivalent to

$$
B^{*} F^{*} S^{T}(s) \psi=0, \quad 0 \leqslant s \leqslant t
$$

Now we obtain from (3) that $B^{*} F^{*}=B^{*}$, and hence i) follows from the definition of $\mathcal{N}_{t}^{T}$. only if

ii) It follows from (10) that $\psi$ is orthogonal to $\mathscr{R}_{f}$ if and

$$
\psi \perp G F \mathscr{R}_{t-h} \text { and } \psi \perp \operatorname{Im} G D
$$

or equivalently

$$
F^{*} G^{*} \psi \perp \mathscr{R}_{t-h} \text { and } D^{*} G^{*} \psi=0 .
$$

Now ii) follows from i).

Q.E.D.

The following corollary is an immediate consequence of the previous lemma.

Corollary 1.3: For every $\psi \in M^{q}$ we have

i) $F^{*} \psi \perp \mathscr{R} \Leftrightarrow \psi \in \mathscr{N}^{T}$

ii) $\psi \perp \mathscr{R} \Leftrightarrow G^{*} \psi \in \mathscr{N}^{T} \cap \operatorname{ker} D^{*}$.

We close this section with a result concerning the relation between $S^{*}(t)$ and $S^{T}(t)$.

Lemma 1.4: Let $\psi \in M^{q}$. Then $z(t)=\left[S^{*}(t+h) \psi\right]^{0}, t \geqslant-h$, solves $\Sigma^{T}$ for $t \geqslant 0$ with initial condition

$$
\left(z(0), z_{0}\right)=G^{*} \psi
$$

Proof: For $-h \leqslant \tau<0$ we have, by a result of Bernier and Manitius [2],

$$
\begin{aligned}
z(\tau) & =\left[S^{*}(h+\tau) \psi\right]^{0} \\
& =X^{T}(h+\tau) \psi^{0}+\int_{-h}^{0} X^{T}(h+\tau+\sigma) \psi^{1}(\sigma) d \sigma \\
& =\left[G^{*} \psi\right]^{1}(\tau) .
\end{aligned}
$$

Moreover, from (5) and (6) we obtain that

$$
z(t)=\left[S^{*}(t) F^{*} G^{*} \psi\right]^{0}=\left[F^{*} S^{T}(t) G^{*} \psi\right]^{0}=\left[S^{T}(t) G^{*} \psi\right]^{0}
$$

holds for $t \geqslant 0$. This proves the lemma.

Q.E.D.

\section{APProximate NuLl-CONTROLlability}

The delay system $\Sigma$ is said to be approximately null-controllable in time $t$ if for any $\epsilon>0$ there exists a control input $u(\cdot) \in L^{p}\left([0, t] ; \mathbb{R}^{m}\right)$ such that the state $\left(x(t), x_{t}\right) \in M^{p}$ at time $t$ is $\epsilon$ close to zero. System $\Sigma^{T}$ is said to be finally observable in time $t$ if

$$
y(s)=0, \quad 0 \leqslant s \leqslant t \quad \Rightarrow \quad x(s)=0, \quad t-h \leqslant s \leqslant t .
$$

We restate this as follows.

Definition 2.1:

i) $\Sigma$ is said to be approximately null-controllable in time $t$, if $\operatorname{Im} S(t) \subset \operatorname{cl}\left(\mathscr{R}_{\mathrm{r}}\right)$. 
$\operatorname{ker} S^{T}(t)$.

ii) $\Sigma^{T}$ is said to be finally observable in the time $t$, if $\mathscr{N}_{t}^{T} \subset$

Definition 2.2:

i) $\Sigma$ is said to be spectrally controllable if $\Sigma_{\lambda}$ is controllable for every $\lambda \in \sigma(A)$.

ii) $\Sigma^{T}$ is said to be spectrally observable if $\Sigma_{\lambda}^{T}$ is observable for every $\lambda \in \sigma\left(A^{T}\right)$.

Remarks 2.3:

i) It is well known that each of the following conditions is equivalent to spectral controllability of $\Sigma$

$$
\begin{gathered}
\operatorname{rank}\left[\Delta(\lambda) B_{0}\right]=n \quad \forall \lambda \in \sigma(A) \quad \text { (see [3] and [22]), } \\
X_{\lambda} \subset \operatorname{cl}(\mathscr{R}) \quad \forall \lambda \in \sigma(A) .
\end{gathered}
$$

Indeed, the second property follows simply from the facts that the reachable subspace $\mathscr{R}_{\lambda}$ of $\Sigma_{\lambda}$ is given by $\mathscr{R}_{\lambda}=P_{\lambda} \mathscr{R}$ and that $P_{\lambda} \operatorname{cl}(\mathscr{R}) \subset \operatorname{cl}(\mathscr{R})$.

ii) Analogously spectral observability of $\Sigma^{T}$ is equivalent to each of the following conditions:

$$
\begin{aligned}
\operatorname{rank}\left[\begin{array}{c}
\Delta^{T}(\lambda) \\
B_{0}^{T}
\end{array}\right] & =n \quad \forall \lambda \in \sigma\left(A^{T}\right) \quad(\text { see }[3]), \\
X_{\lambda}^{T} \cap \mathscr{N}^{T} & =\{0\} \quad \forall \lambda \in \sigma\left(A^{T}\right) .
\end{aligned}
$$

Throughout this section let $T_{0}$ denote the exponential growth of the entire matrix function

$$
\operatorname{adj} \Delta(s) B_{0}, \quad s \in \mathbb{C} .
$$

Note that $T_{0}$ is always less than or equal to $(n-1) h$. Then Olbrot [21, Lemma 1] has shown by the use of Laplace transform methods that

$$
\mathscr{N}^{T}=\mathscr{N}_{t}^{T} \quad \text { for } t>T_{0}+h .
$$

This result can be slightly improved.

Lemma 2.4: For every $t>T_{0}$ we have

$$
\mathscr{N}^{T} \cap \operatorname{ker} D^{*}=\mathscr{N}_{t}^{T} \cap \operatorname{ker} D^{*} .
$$

Proof: Let $t_{1}>T_{0}$ be fixed and let $z(t), t \geqslant-h$, be a solution of $\Sigma^{T}$ with initial condition

$$
\left(z(0), z_{0}\right)=\psi \in \mathscr{N}_{t_{1}}^{T} \cap \operatorname{ker} D^{*} .
$$

Then the corresponding output function

$$
w(t)=B_{0}^{T} z(t-h)
$$

vanishes for $0 \leqslant t \leqslant t_{1}+h$. Moreover, some straightforward manipulations show that the Laplace transform $\hat{w}(s)$ of $w(t)$ satisfies

$$
\operatorname{det} \Delta(s) \cdot \hat{w}(s)=B_{0}^{T} \operatorname{adj} \Delta^{T}(s) \Psi(s)
$$

where

$$
\begin{aligned}
\Psi(s)= & e^{-s h} \psi^{0}+s \int_{-h}^{0} e^{-s(h+\tau)} \psi^{1}(\tau) d \tau \\
& -\int_{-h}^{0} d \eta^{T}(\tau) \int_{-h}^{\tau} e^{-s(h+\sigma-\tau)} \psi^{1}(\sigma) d \sigma
\end{aligned}
$$

(compare [13, eq. (3.4)] and [10, eq. (6)]). Since $\Psi(s)$ is an entire function of exponential growth less than or equal to $h$, we can now proceed as in the proof of Olbrot [21, Lemma 1] and obtain that $w(t)$ vanishes identically. Hence, $\psi \in \mathscr{N}^{T} \cap \operatorname{ker} D^{*}$ and the lemma is proved.

Q.E.D.

Combining Lemma 1.2 and Lemma 2.4, we obtain the following corollary.

Corollary 2.5: For every $t>T_{0}+h$ we have

$$
\operatorname{cl}(\mathscr{R})=\operatorname{cl}\left(\mathscr{R}_{t}\right), \quad \mathscr{N}^{T}=\mathscr{N}_{t}^{T} .
$$

In particular this holds for every $t>n h$.

Note that for systems with single point delays it is already known [1, Corollary 5.1] that $\mathscr{R}_{t}=\mathscr{R}$ for $t>n h$.

Now let us define $T_{1} \geqslant 0$ to be the minimal time satisfying

$$
\operatorname{ker} S^{T}\left(T_{1}\right)=\operatorname{ker} S^{T}(t) \quad \forall t \geqslant T_{1} .
$$

Such a time $T_{1}$ exists by Henry [10] and is always less than or equal to $n h$ (see also Kappel [13, Theorem 3.1]).

Lemma 2.6: For every $t \geqslant T_{1}$ the following statements are equivalent:

i) $\mathscr{N}^{T} \subset \operatorname{ker} S^{T}(t)$

ii) $\operatorname{Im} S(t) \subset \operatorname{cl}(\mathscr{R})$.

Proof: First let $\mathscr{N}^{T} \subset \operatorname{ker} S^{T}(t)$. Then, by Corollary 1.3 ii), $\psi \perp \mathscr{R}$ implies $G^{*} \psi \in \mathscr{N}^{T}$, and hence $S^{T}(t) G^{*} \psi=0$. Now, by (5), we have $G^{*} S^{*}(t) \psi=0$, and thus $S^{*}(t) \psi=0$, since $G^{*}$ is injective. This shows that $\psi$ is orthogonal to $\operatorname{Im} S(t)$, and ii) holds.

Conversely, let $\operatorname{Im} S(t) \subset c l(\mathscr{R})$. Then, by Corollary $1.3 \mathrm{i}$ ), $\psi \in \mathscr{N}^{T}$ implies $F^{*} \psi \perp \mathscr{R}$ and thus $F^{*} \psi \perp \operatorname{Im} S(t)$. Applying (5) and (6), we obtain

$$
S^{T}(t+h) \psi=G^{*} F^{*} S^{T}(t) \psi=G^{*} S^{*}(t) F^{*} \psi=0 .
$$

We conclude that $S^{T}(t) \psi=0$, since $t \geqslant T_{1}$. This proves i). Q.E.D

Remark 2.7: Note that the assumption $t \geqslant T_{1}$ was not needed for the implication i) $\Rightarrow$ ii).

Now we are in the position to prove the main result of this section.

Theorem 2.8: Let $t>T_{0}+h$ and $t \geqslant T_{1}$. Then the following statements are equivalent:

i) $\Sigma$ is spectrally controllable,

ii) $\Sigma$ is approximately null-controllable in time $t$,

iii) $\Sigma^{T}$ is spectrally observable,

iv) $\Sigma^{T}$ is finally observable in time $t$.

In particular the above equivalence holds for every $t>n h$.

Proof: Clearly, i) and iii) are equivalent by the well-known rank conditions for spectral controllability and observability.

The equivalence of ii) and iv) follows from Lemma $2.6\left(t \geqslant T_{1}\right)$ together with Corollary $2.5\left(t>T_{0}+h\right)$.

Hence, it remains to prove that i) and ii) are equivalent. In fact, $\Sigma$ is spectrally controllable if and only if

$$
X_{\lambda} \subset \operatorname{cl}(\mathscr{R}) \quad \forall \lambda \in \sigma(A) .
$$

Now it has been shown in [15, Lemma 4.3] that, for $t \geqslant T_{1}$, we have

$$
c l(\operatorname{Im} S(t))=c l\left(\operatorname{span}\left\{X_{\lambda} \mid \lambda \in \sigma(A)\right\}\right) .
$$

Hence, (14) holds if and only if

$$
\operatorname{Im} S(t) \subset \operatorname{cl}(\mathscr{R}) .
$$

Since $t>T_{0}+h$, this is equivalent to approximate null-controllability of $\Sigma$ in time $t$ (Corollary 2.5).

Remarks 2.9:

i) Note that the equivalence of spectral observability and final observability has already been proved by Olbrot [21, Proposition 2]. 
ii) The equivalence of spectral controllability and approximate null-controllability has been proved in [23] for the special case of a single point delay.

\section{APPROXIMATE CONTROLLABILITY AND OBSERVABILITY}

In the beginning of this section let us recall some definitions. Definition 3.l: $\Sigma$ is said to be:

i) approximately controllable, if $\operatorname{cl}(\mathscr{R})=M^{p}$,

ii) approximately $F$-controllable, if $c l(F \mathscr{R})=c l(\operatorname{lm} F)$.

The following definition gives the corresponding dual observability notions for $\Sigma^{T}$.

Definition 3.2: $\Sigma^{T}$ is said to be

i) strictly observable, if $\mathscr{N}^{T} \cap \operatorname{ker} D^{*}=\{0\}$, i.e.,

$$
y(t)=0 \quad \forall t \geqslant-h \quad \Rightarrow \quad z(t)=0 \quad \forall t \geqslant-h,
$$

ii) observable, if $\mathscr{N}^{T}=\operatorname{ker} F^{*}$, i.e.,

$$
y(t)=0 \quad \forall t \geqslant 0 \quad \Rightarrow \quad z(t)=0 \quad \forall t \geqslant 0 .
$$

Manitius has proved in [17] that $\Sigma$ is approximately $F$-controllable if and only if $\Sigma^{T}$ is observable in the sense of Definition 3.2 ii). Note that this equivalence also follows directly from Corollary 1.3 i). Moreover, Manitius has given two necessary conditions for approximate $F$-controllability. In the following we show that these conditions together are also sufficient.

Theorem 3.3: The following statements are equivalent:

i) $c l(F \mathscr{R})=c l(\operatorname{Im} F)$,

ii) $\Sigma$ is spectrally controllable and

$$
\operatorname{ker} F^{*} G^{*} \cap \operatorname{ker} D^{*} G^{*} \cap \operatorname{Im} F^{*}=\{0\},
$$

iii) $\mathscr{N}^{T}=\operatorname{ker} F^{*}$.

Proof:

i) $\Leftrightarrow$ iii): First note that in any case we have

$$
\operatorname{ker} F^{*} \subset \mathscr{N}^{T} \text {. }
$$

Hence iii) holds if and only if

$$
\psi \in \mathscr{N}^{T} \Rightarrow F^{*} \psi=0
$$

which, by Corollary 1.3 i), is equivalent to the implication

$$
F^{*} \psi \perp \mathscr{R} \Rightarrow F^{*} \psi=0 .
$$

Finally, this is equivalent to i).

i) $\Rightarrow$ ii): This has been proved in [17]. Here we present an alternative proof.

First let $\psi \perp \mathscr{R}$. Then, since $G F \mathscr{R}=S(h) \mathscr{R} \subset \mathscr{R}$, it follows that $F^{*} G^{*} \psi \perp \mathscr{R}$. By i), this implies $F^{*} G^{*} \psi=0$ or equivalently, $\psi \perp$ $\operatorname{Im} G F=\operatorname{Im} S(h)$. We conclude that $\Sigma$ is approximately null-controllable, and hence, by Theorem 2.8 , spectrally controllable.

Second, let $F^{*} G^{*} F^{*} \psi=0$ and $D^{*} G^{*} F^{*} \psi=0$ for some $\psi \in M^{q}$. Then $F^{*} \psi \perp \operatorname{Im} G F$ and $F^{*} \psi \perp \operatorname{Im} G D$. By (10), this implies $F^{*} \psi$ $\perp \mathscr{R}$, and hence $F^{*} \psi=0$. This proves (15).

ii) $\Rightarrow$ ij: First of all ii) implies approximate null-controllability of $\Sigma$ in time $t=(n+1) h$ (Theorem 2.8), i.e.,

$$
\operatorname{Im}(G F)^{n+1}=\operatorname{Im} S((n+1) h) \subset \operatorname{cl}(\mathscr{R}) .
$$

Now let $F^{*} \psi \perp \mathscr{R}$. Then $F^{*} \psi$ is orthogonal to $\operatorname{Im}(G F)^{n-1}$, and hence $\left(F^{*} G^{*}\right)^{n+1} F^{*} \psi=0$.

Define

$$
\psi_{k}=\left(F^{*} G^{*}\right)^{k} F^{*} \psi, \quad k=0,1, \cdots, n+1 .
$$

then, by induction, we show that $\psi_{k}=0$ for $k=n+1, \cdots, 0$. In fact $\psi_{n+1}=0$. Moreover, $\psi_{k}=0,1 \leqslant k \leqslant n+1$, implies

$$
\begin{aligned}
& \psi_{k-1} \in \operatorname{ker} F^{*} G^{*} \\
& \psi_{k-1} \in \operatorname{Im} F^{*} \\
& \psi_{k-1} \in \operatorname{ker} D^{*} G^{*}
\end{aligned}
$$

where the latter statement follows from the fact that $\psi_{k-1}$ is orthogonal to $\mathscr{R}$ and that $\operatorname{Im} G D \subset \mathscr{R}$ (Lemma 1.1). Hence, by (15), $\psi_{k-1}=0$. This shows that $F^{*} \psi=\psi_{0}=0$.

Remarks 3.4:

i) A solution of system $\Sigma^{T}$ is said to be small [10] if it vanishes after some finite time $T \geqslant-h$. A small solution is called trivial [15] if it vanishes for $t \geqslant 0$.

ii) Since $S^{T}(h)=G^{*} F^{*}$, condition (15) is equivalent to the property

$$
\left.\begin{array}{ll}
y(t)=0 & \forall t \geqslant 0 \\
x(t)=0 & \forall t \geqslant h
\end{array}\right\} \Rightarrow x(t)=0 \quad \forall t \geqslant 0
$$

for the solutions of system $\Sigma^{T}$. This property is called observability of the nontrivial small solutions [26], [27], since it means that every small solution of $\Sigma^{T}$ with zeto output is trivial.

The following theorem-which is strictly analogous to Theorem 3.3-contains a duality result for approximate controllability (namely the equivalence of conditions i) and iii) below) which is apparently new. Ther remainder of the theorem is a generalization of a result in [16].

Theorem 3.5: The following statements are equivalent:

i) $\mathscr{R}$ is dense in $M^{P}$,

ii) $\Sigma$ is spectrally controllable and

$$
\operatorname{ker} F^{*} \cap \operatorname{ker} D^{*}=\{0\},
$$

iii) $\mathscr{N}^{T} \cap \operatorname{ker} D^{*}=\{0\}$.

Proof:

i) $\Rightarrow$ ii): i) implies $F$-controllability, and hence, by Theorem 3.3 , spectral controllability of $\Sigma$.

For (16) see [16, Theorem 1].

ii) $\Rightarrow$ iii): Since (16) is stronger than (15), it follows from Theorem 3.3 that ii) implies observability of $\Sigma^{T}$, i.e., $\mathscr{N}^{T}=$ ker $F^{*}$. Now iii) follows directly from (16).

iii) $\Rightarrow$ i): Let $\mathscr{N}^{T} \cap \operatorname{ker} D^{*}=\{0\}$ and $\psi \perp \mathscr{R}$. Then, by Corollary $1.3 \mathrm{ii)}, G^{*} \psi \in \mathscr{N}^{T} \cap \operatorname{ker} D^{*}$, and thus $G^{*} \psi=0$. Since $G^{*}$ is injective, it follows that $\psi=0$. Hence, $\mathscr{R}$ is dense in $M^{p}$.

\section{Remarks 3.6:}

Q.E.D.

i) The respect in which Theorem 3.5 generalizes the result in [16] is that Manitius considers only functions $\eta(\tau)$ of bounded variation containing no singular part and having only finitely many jumps. We mention also that our proof is simpler and more direct than that appearing in [16].

ii) Condition (16) is equivalent to the property

$$
\left.\begin{array}{ll}
y(t)=0 & \forall t \geqslant-h \\
x(t)=0 & \forall t \geqslant 0
\end{array}\right\} \Rightarrow x(t)=0 \quad \forall t \geqslant-h
$$

for the solutions of system $\Sigma^{T}$. This property is called observability of the small solutions [26] since it means that every nonzero small solution of $\Sigma^{T}$ has a nonzero output.

iii) If $\eta(\tau)=\eta(-h)+A_{1}$ on the interval $(-h, \epsilon-h]$ for some $\epsilon>0$, then condition (16) is equivalent to

$$
\operatorname{rank}\left[\begin{array}{ll}
A_{1} & B_{0}
\end{array}\right]=n
$$

(see [16] or [26]).

We have shown in Theorem 3.3 that approximate $F$-controllability is equivalent to a) spectral controllability and b) observability of the nontrivial small solutions of the transposed system. In 
Remark 2.3 we have mentioned a matrix type criterion for spectral controllability. We conclude this section by stating a verifiable matrix criterion for observability of the nontrivial small solutions for systems with commensurate delays.

Theorem 3.7: Let L: $\mathscr{C} \rightarrow \mathbb{R}^{n}$ be given by (1). Then the nontrivial small solutions of system $\Sigma^{T}$ are observable if and only if the following equation holds for some $\lambda \in \mathbb{C}$

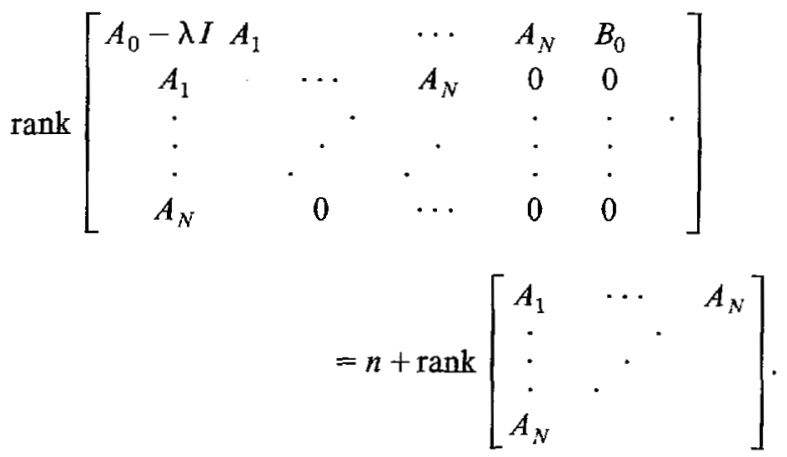

Proof: First note that the nontrivial small solutions of system $\Sigma^{T}$ are observable if and only if

$$
\left.\begin{array}{ll}
y(t)=0 & \forall t \geqslant 0 \\
x(t)=0 & \forall t \geqslant \alpha
\end{array}\right\} \Rightarrow x(t)=0 \quad \forall t \geqslant 0 .
$$

This follows easily by induction.

Second, let us define

$$
f_{j}(t):=x(t-j \alpha), \quad 0 \leqslant t \leqslant \alpha, \quad j=1, \cdots, N,
$$

for any function $x(t), t \geqslant-h=-N \alpha$.

Then $x(t)$ is a solution of $\Sigma^{T}$ which vanishes for $t \geqslant \alpha$ if and only if the equations

$$
\begin{aligned}
\dot{x}(t) & =A_{0}^{T} x(t)+\sum_{j=1}^{N} A_{j}^{T} f_{j}(t), \\
0 & =A_{k}^{T} x(t)+\sum_{j=k+1}^{N} A_{j}^{T} f_{j-k}(t)
\end{aligned}
$$

hold for $k=1, \cdots, N$ and $0 \leqslant t \leqslant \alpha$. Hence, (19) is satisfied if and only if the finite dimensional system

$$
\begin{aligned}
\dot{x}(t) & =A_{0}^{T} x(t)+\sum_{j=1}^{N} A_{j}^{T} f_{j}(t), \\
y_{k}(t) & =A_{k}^{T} x(t)+\sum_{j=k+1}^{N} A_{j} f_{j-k}(t), \quad k=1, \cdots, N, \\
y_{0}(t) & =B_{0}^{T} x(t)
\end{aligned}
$$

has the property that $y_{k}(t)=0$ for $0 \leqslant t \leqslant \alpha, k=0, \cdots, N$, and $x(\alpha)=0$ imply $x(t)=0$ for $0 \leqslant t \leqslant \alpha$ for any control input $f_{j}(\cdot) \in L^{p}\left([0, \alpha] ; \mathbb{R}^{m}\right), j=1, \cdots, N$. This means that system $(20)$ is input-observable in the sense of [26, Appendix]. By [26, Theorem A6], this is equivalent to condition (18).

Q.E.D.

In the special case of a single point delay $(N=1)$ condition (18) reduces to

$$
\operatorname{rank}\left[\begin{array}{ccc}
A_{0}-\lambda I & A_{1} & B_{0} \\
A_{1} & 0 & 0
\end{array}\right]=n+\operatorname{rank} A_{1}
$$

for some $\lambda \in \mathbb{C}$. This is precisely the criterion which has been derived by Manitius [17]. A generalization to neutral systems can be found in the authors paper [27].

\section{EXACT NULL-CONTROLLABILITY AND CONTINUOUS FINAL OBSERVABILITY}

Throughout this section we denote by $z(t)=z(t, \psi), t \geqslant-h$, the unique solution of $\Sigma^{T}$ corresponding to the initial condition $z(0)=\psi^{0}, z(\tau)=\psi^{1}(\tau),-h \leqslant \tau<0$, where $\psi \in M^{q}$. This means that $z(t, \psi)$ is given by

$$
z(t, \psi)=\left\{\begin{array}{l}
{\left[S^{T}(t) \psi\right]^{0}, \quad t \geqslant 0,} \\
\psi^{1}(t), \quad-h \leqslant t \leqslant 0 .
\end{array}\right.
$$

Again we start with definitions. System $\Sigma$ is said to be exactly null-controllable if there exists a time $T>0$ such that for every initial state $\varphi \in M^{p}$ there exists a control $u(\cdot) \in L^{p}\left([0, T] ; \mathbb{R}^{m}\right)$ such that the corresponding solution $x(t), t \geqslant-h$, satisfies $x(t)$ $=0$ for $T-h \leqslant t \leqslant T$. Alternatively, we can state this as follows.

Definition 4.1: System $\Sigma$ is said to be exactly null-controllable if there exists some $T>0$ such that $\operatorname{Im} S(T) \subset \mathscr{R}_{T}$.

Remarks 4.2:

i) If $\Sigma$ is exactly null-controllable, then the time $T$ in the above definition must be greatear than $h$.

ii) In [1] a criterion has been given for exact null-controllability in an arbitrary short time $(T>h)$. In [11] it has been shown that exact null-controllability in the sense of the above definition (in an arbitrary large time) is equivalent to spectral controllability if $\Sigma$ is a two-dimensional system with a single point delay. In general, this problem is, we judge, still open (we mention that this equivalence is claimed by Marchenko [19]; however, the arguments in [19] seem incomplete).

In this paper we show that the exact null-controllability of $\Sigma$ is dual to the following observability notion for $\Sigma^{T}$.

Definition 4.3: System $\Sigma^{T}$ is said to be continuously finally observable if there exists some $T>0$ and $K>0$ such that the following inequality holds for every $\psi \in M^{q}$ :

$$
\left\|S^{T}(T) \psi\right\|_{M^{q}} \leqslant K\left\|B_{0}^{T} z(\cdot, \psi)\right\|_{L^{q}\left([0, T] ; \mathbf{R}^{m}\right)} .
$$

Remark 4.4: If system $\Sigma^{T}$ is continuously finally observable, then from the fact that the output $y(\cdot)$ is small in the sense of the $L^{q}$-norm on $[0, T]$ one can conclude that the system state at time $T$ is small in the $M^{q}$-norm. This means that the final state can be reconstructed from the output on the interval $[0, T]$ by means of a continuous, linear operator. Such a property is of particular interest for the design of an exact observer.

The main step towards our duality result is given by the following lemma.

Lemma 4.5: The following statements are equivalent:

i) $\operatorname{Im} S(T) \subset \mathscr{R}_{T}$.

ii) There exists some $K>0$ such that for every $\psi \in M^{q}$

$$
\left\|F^{*} S^{T}(T-h) \psi\right\|_{M^{q}} \leqslant K\left\|B_{0}^{T} z(\cdot, \psi)\right\|_{L^{q}\left([-h, T-h] ; \mathbb{R}^{m}\right)} .
$$

Proof: i) holds if and only if there exists some $K>0$ such that for all $\psi \in M^{q}$ the following inequality holds

$$
\left\|S^{*}(T) \psi\right\|_{M^{q}} \leqslant K\left\|B^{*} S^{*}(\cdot) \psi\right\|_{L^{q}\left([0, T] ; \mathbb{P}^{m}\right)}
$$

(see Dolecki and Russell [7, Theorem 2.3]). Now, by Lemma 1.4, we have for every $t \in[0, T]$

$$
B^{*} S^{*}(t) \psi=B_{0}^{T}\left[S^{*}(t) \psi\right]^{0}=B_{0}^{T} z\left(t-h, G^{*} \psi\right),
$$

and moreover, by (5) and (6),

$$
S^{*}(T) \psi=S^{*}(T-h) F^{*} G^{*} \psi=F^{*} S^{T}(T-h) G^{*} \psi .
$$

Hence, (24) is equivalent to the following inequality:

$$
\left\|F^{*} S^{T}(T-h) G^{*} \psi\right\|_{M^{q}} \leqslant K\left\|B_{0}^{T} z\left(\cdot, G^{*} \psi\right)\right\|_{L^{q}\left([-h, T-h] ; \mathbb{R}^{m}\right)} .
$$


Note that this is nothing else than (23) with $\psi$ replaced by $G^{*} \psi$. Since $\operatorname{Im} G^{*}=\left\{\psi \in M^{q} \mid \psi^{1} \in W^{1, q}, \psi^{0}=\psi^{1}(0)\right\}$ is dense in $M^{q}$, the lemma is proved.

Now we are able to prove the desired duality result.

Theorem 4.6: $\Sigma$ is exactly null-controllable if and only if $\Sigma^{T}$ is continuously finally observable.

Proof: Let $\Sigma$ be exactly null-controllable in time $T$. Then $T>h$ and, by Lemma 4.5, there exists some $K>0$ such that (23) holds for all $\psi \in M^{q}$. Hence, we have for every $\psi \in M^{q}$

$$
\begin{aligned}
& \left\|S^{T}(T+h) \psi\right\|_{M^{q}} \\
& \quad \leqslant\left\|G^{*}\right\| \cdot\left\|F^{*} S^{T}(T-h) S^{T}(h) \psi\right\|_{M^{q}} \\
& \quad \leqslant\left\|G^{*}\right\| \cdot K \cdot\left\|B_{0}^{T z}\left(\cdot, S^{T}(h) \psi\right)\right\|_{L^{q}\left([-h, T-h]: \mathbb{R}^{m}\right)} \\
& \quad=\left\|G^{*}\right\| \cdot K \cdot\left\|B_{0}^{T} z(\cdot, \psi)\right\|_{L^{q}\left([0, T] ; \mathbb{R}^{m}\right)} \\
& \quad \leqslant\left\|G^{*}\right\| \cdot K \cdot\left\|B_{0}^{T} z(\cdot, \psi)\right\|_{L^{q}\left([0, T \div h] ; \mathbf{R}^{m}\right)}
\end{aligned}
$$

and $\Sigma^{T}$ is continuously finally observable at time $T+h$.

Conversely continuous final observability of $\Sigma^{T}$ at time $T$ implies the existence of a $K>0$ such that (22) holds for all $\psi \in M^{q}$. Hence, we have for every $\psi \in M^{q}$

$$
\begin{aligned}
& \left\|F^{*} S^{T}(T) \psi\right\|_{M^{q}} \\
& \quad \leqslant\left\|F^{*}\right\| \cdot\left\|S^{T}(T) \psi\right\|_{M^{q}} \\
& \quad \leqslant\left\|F^{*}\right\| \cdot K \cdot\left\|B_{0}^{T} z(\cdot, \psi)\right\|_{L^{q}\left([0, T]: \mathbb{R}^{m}\right)} \\
& \quad \leqslant\left\|F^{*}\right\| \cdot K \cdot\left\|B_{0}^{T} z(\cdot, \psi)\right\|_{L^{q}\left([-h, T] ; \mathbb{R}^{m}\right)} .
\end{aligned}
$$

By Lemma 4.5 this implies that $\Sigma$ is exactly null-controllable at time $T+h$. Q.E.D.

In fact, we have proved a slightly more refined result than the assertions of Theorem 4.6, namely exact null-controllability of $\Sigma$ in time $T$ implies continuous final observability of $\Sigma^{T}$ in time $T+h$ and continuous final observability of $\Sigma^{T}$ in time $T$ implies exact null-controllability of $\Sigma$ in time $T+h$.

\section{CONCLuSions}

In this paper we have filled several gaps in the existing literature on retarded functional differential systems concerning the relations between the various notions of controllability and observability in the product space $\mathbb{R}^{n} \times L^{p}$. In particular, we have shown that spectral controllability is equivalent to approximate null-controllability respectively final observability of the transposed system. The significance of this result follows from the fact that spectral controllability implies feedback stabilizability with an arbitrary exponential decay rate and can be characterized by a matrix type condition. Moreover, we have shown that approximate $F$-controllability is equivalent to a) spectral controllability and b) observability of the nontrivial small solutions for the transposed system, and we have derived a verifiable matrix type criterion for $b$ ) in the case of commensurate delays. This generalizes and extends recent results of Manitius [17].

Finally, we have shown that a retarded functional differential system is exactly null-controllable if and only if the transposed system is continuously finally observable. There remains the open question if spectral controllability is equivalent to exact null-controllability for general linear, retarded systems.

\section{ACKNOWLEDGMENT}

The author wishes to thank Dr. R. B. Vinter and Dr. A. Manitius for some helpful comments.

\section{REFERENCES}

[1] H. T. Banks, M. Q. Jacobs, and C. E. Langenhop, "Characterization of the controlled states in $W_{2}^{(i)}$ of linear hereditary systems," SIAM J.Contr., vol. 13, pp. 611-649, 1975.

[2] C. Bernier and A. Manitius, "On semigroups in $\mathbb{R}^{n} \times L^{p}$ corresponding to differential equations with delays," Can. J. Math., vol. 30, pp. 897-914, 1978.

[3] K. P. M. Bhat and H. N. Koivo, "Modal characterization of controllability and observability for time delay systems," IEEE Trans. Automat. Contr., vol. AC-21, pp. 292-293, 1976.

[4] K. P. M. Bhat and H. N. Koivo, "An observer theory for time delay systems," IEEE Trans. Automat. Contr., vol. AC-21, pp. 266-269, 1976.

[5] M. C. Delfour, "The largest class of hereditary systems defining a $C_{0}$-semigroup on the product space," Can. J. Math., vol. 32 , pp. 969-978, 1980

[6] M. C. Delfour and A. Manitius, "The structural operator $F$ and its role in the theory of retarded systems," Part I, J. Math. Anal. Appl., vol. 73, pp. 466-490, 1980; Part II, J. Math. Anal. Appl., vol. 74 , pp. $359-381,1980$.

[7] S. Dolecki and D. L. Russell. "A general theory of observation and control," SIAM J. Contr. Opt., vol. 15, pp. 185-220, 1977.

[8] H. O. Fattorini, "Some remarks on complete controllability," SIAM J. Conir., vol. 4, pp. 686-694, 1966.

[9] J. Hale, Theory of Functional Differential Equations. New York: Springer-Verlag, 1977

[10] D. Henry, "Small solutions of linear autonomous functional differential equations," J. Diff. Equations, vol. 8, pp. 494-501, 1970.

[11] M. Q. Jacobs and C. E. Langenhop, "Controllable two dimensional neutral systems," in Mathematical Systems Theory, vol. 1, S. Dolecki, C. Olech, and J. Zabczyk, Eds. Warsaw, Poland: Banach Center Publications, 1976.

[12] E. W. Kamen, "An operator theory of linear functional differential equations," J. Diff. Equations, vol. 27, pp. 274-297, 1978.

[13] F. Kappel, "Laplace transform methods and linear autonomous functional differential equations," Math. Institut, University of Graz, Bericht 64, 1976

[14] A. Manitius, "Controllability, observability and stabilizability of retarded systems," in Proc. 1976 IEEE Conf. on Decision and Contr., New York, 1976, pp. 752-758.

[15] __ "Completeness and F-completeness of eigenfunctions associated with retarded functional differential equations," $J$. Diff. Equations, vol. $35, \mathrm{pp} .1-29,1980$.

[16] _- "Necessary and sufficient conditions of approximate controllability for linear retarded systems," SIAM J. Contr. Opt., vol. 19. pp. 516-532, 1981.

[17] - "F-controllability and observability of linear retarded systems," Appl. Math. Opt., vol. 9, pp. 73-95, 1982.

[18] A. Manitius and R. Triggiani, "Function space controllability of linear retarded systems: $\AA$ derivation from abstract operator conditions," SIAM J. Contr. Opt., vol. 16, pp. 599-645, 1978.

[19] V. M. Marchenko, "On complete controllability of systems with delay," Prob. Contr. Inform. Theory, vol. 8, pp. 421-432, 1979.

[20] A. S. Morse, "Ring models for delay differential systems," Automatica, vol. 12, pp. 529-531, 1976.

[21] A. W. Olbrot, "Observability and observers for a class of linear systems with delays," IEEE Trans. Automat. Contr., to be published.

[22] L. Pandolfi. "Feedback stabilization of functional differential equations," Bollettino U.M.I., vol. 12, pp. 626-635, 1975.

[23] K. M. Przyluski, "Infinite dimensional discrete time systems: Theory and applications to linear hereditary control systems," Warsaw, Poland, Tech. Rep., 1979.

[24] D. Salamon, "Observers and duality between observation and state feedback for time delay systems," IEEE Trans. Automat. Contr., vol. AC- 25 , pp. $1187-1192,1980$.

[25] - "On dynamic observation and state feedback for time delay systems," in Evolution Equations and Their Applications (Research Notes in Mathematics), vol. 68, F. Kappel and W. Schappacher, Eds. 1981, London: Pitman, 1982, pp. 202-219.

[26] - "Control and observation of neutral systems," Research Notes in Mathematics, vol. 91, London: Pitman, 1984.

[27] _- "Observability of nontrivial small solutions for neutral systems," Syst. Contr. Lett., vol. 3. pp. 91-99, 1983. 
[28] E. D. Sontag, "Linear systems over commutative rings: A survey," Ric. di Automatica, vol. 7, pp. 1-34, 1976.

[29] R. B. Vinter, "Semigroups on product spaces with applications to initial value problems with nonlocal boundary conditions," in Control of Distributed Parameter Systems, S. P. Banks and A. J. Pritchard, Eds. New York: Pergamon, 1978, pp. 91-98.

Dietmar Salamon was born on March 7, 1953, in Bremen, West Germany. He received the Diploma in mathematics in 1977 and the

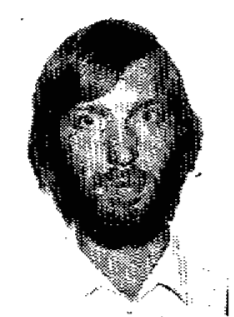

Ph.D. degree in 1982 , both from the University of Bremen, Bremen, West Germany.

From 1978 to 1983 he was with the Forschungsschwerpunkt "Dynamische Systeme" at the University of Bremen. At present he is Research Associate at the Mathematics Research Center, University of Wisconsin, Madison. His main fields of interest include structure theory and approximation of functional differential equations, control theory, infinite dimensional linear systems theory, and linear quadratic optimal control.

\section{Technical Notes and Correspondence}

\section{Root Locus Near Isolated Pole-Zero Dipoles and a Counterintuitive Case of Digital Control Compensation}

D. A. PIERRE

Abstract - A convenient characterization is given for root loci associated with open-loop pole-zero dipoles. A particular dipole-locus effect is shown to influence the design of a digital PID controller for an oscillatory plant; the design is a counterintuitive one in which zeros of the compensator are placed outside the unit circle in the neighborhood of the plant poles.

\section{INTRODUCTION}

When open-loop transfer functions contain complex conjugate poles near the stability boundary, it is common ([1], for example) to have controller zeros specified in the neighborhood of the poles. Pole-zero pairs or dipoles invariably result in such cases because of plant uncertainty and drift. The question of plant uncertainty is one that must be addressed in all designs, but is especially important in those cases where plant poles occur near the stability boundary. When dipoles occur inside the stable region, the effects of dipole "hidden modes" approach zero as the separation between the pole and zero in question approaches zero [2]. When dipoles occur on or very near the stability boundary, however, the "hidden modes" may cause stability problems if not properly anticipated.

Given is a pole-zero pair (Fig. 1) which is assumed to be part of an open-loop transfer function $K G(z)$ where $K$ is a real value and $G(z)$ is a rational fraction in the complex variable $z$. We are interested in the case where other poles and zeros of $G(z)$ are far removed from the pole-zero pair, in which case the distance $2 \epsilon$ of Fig. 1 is much smaller than the distance between the pole-zero pair and any other pole or zero of $G(z)$. Such an isolated pole-zero pair is called a dipole.

When the net phase angle contributed by other open-loop poles and zeros is assumed constant in the neighborhood of a pole-zero pair, the resulting dipole root-locus branch is an arc of a circle. Horowitz [3] constructs the arc-of-circle dipole locus by using calculated angles of

Manuscript received February 11, 1983; revised August 19.1983.

The author is with the Department of Electrical Engineering and Conputer Science, Montana State University, Bozeman, MT 59717

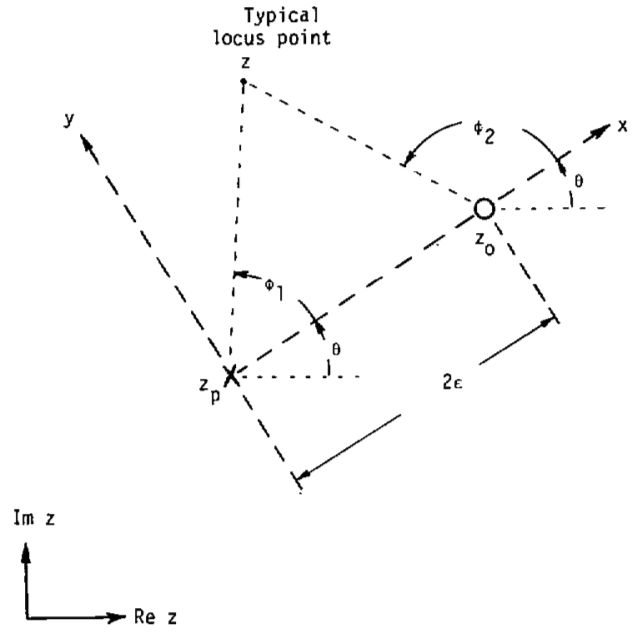

Fig. 1. Pole-zero dipole.

departure and arrival in a particular case where the dipole is on the $j \omega$ axis of the $s$ plane. In the following, Section II, a convenient characterization is given for general arc-of-circle loci [4] of ideal dipoles.

In Section III, a compensator is developed for a particular oscillatory plant [5]. The resulting compensator is counterintuitive in the sense that the placement of compensator zeros for good performance is opposite to what generally would be expected, but is consistent with pole-zero dipole locus theory.

\section{Dipole NeighBorhood Locus}

Closed-loop poles are characterized by

$$
1+K G(z)=0
$$

and the locus of points which satisfy (1) when $K>0$ are governed by

$$
\angle G(z)=-(1+2 m) 180^{\circ}, \quad \text { integer } m
$$

